\title{
A stochastic recursive optimal control problem under the G-expectation framework
}

\author{
Mingshang $\mathrm{Hu}^{*} \quad$ Shaolin $\mathrm{Ji}^{\dagger} \quad$ Shuzhen Yang ${ }^{\ddagger}$
}

\begin{abstract}
In this paper, we study a stochastic recursive optimal control problem in which the objective functional is described by the solution of a backward stochastic differential equation driven by $G$-Brownian motion. Under standard assumptions, we establish the dynamic programming principle and the related Hamilton-Jacobi-Bellman (HJB) equation in the framework of $G$-expectation. Finally, we show that the value function is the viscosity solution of the obtained HJB equation.
\end{abstract}

Key words. G-expectation, backward stochastic differential equations, stochastic optimal control, dynamic programming principle, viscosity solution

AMS subject classifications. 93E20, 60H10, 35K15

\section{Introduction}

It is well known that the nonlinear backward stochastic differential equation (BSDE) was first introduced by Pardoux and Peng [19]. Independently, Duffie and Epstein [5] presented a stochastic differential recursive utility which corresponds to the solution of a particular BSDE. Then the BSDE point of view gives a simple formulation of recursive utilities (see [] $]$ ).

Since then, the classical stochastic optimal control problem is generalized to a so called "stochastic recursive optimal control problem" in which the cost functional is described by the solution of BSDE. Peng 28] obtained the Hamilton-Jacobi-Bellman equation for this kind of problem and proved that the value function is its viscosity solution. In [29], Peng generalized his results and originally introduced the notion of stochastic backward semigroups which allows him to prove the dynamic programming principle in a very straightforward way. This backward semigroup approach is proved to be a useful tool for the stochastic optimal control problems. For instance, Wu and Yu 31] adopted this approach to study one kind of stochastic recursive optimal control problem with the cost functional described by the solution of a reflected BSDE. It is also introduced in the theory of stochastic differential games by Buckdahn and Li in [1]. We emphasize

\footnotetext{
*School of Mathematics, Shandong University, humingshang@sdu.edu.cn. Research supported by the National Natural Science Foundation of China (11201262)

${ }^{\dagger}$ Qilu Institute of Finance, Shandong University, jsl@sdu.edu.cn. Hu, Ji, and Yang's research was partially supported by NSF of China No. 10921101; and by the 111 Project No. B12023.

$\ddagger$ School of mathematics, Shandong University, Jinan, Shandong 250100, PR China. yangsz@mail.sdu.edu.cn. This paper was submitted to SIAM Journal on Control and Optimization on March 5, 2013 (Submitted manuscript No. 091225$.
} 
that Buckdahn et al. 2] obtained an existence result of the stochastic recursive optimal control problem.

Motivated by measuring risk and other financial problems with uncertainty, Peng [22] introduced the notion of sublinear expectation space, which is a generalization of probability space. As a typical case, Peng studied a fully nonlinear expectation, called $G$-expectation $\hat{\mathbb{E}}[\cdot]$ (see [25] and the references therein), and the corresponding time-conditional expectation $\hat{\mathbb{E}}_{t}[\cdot]$ on a space of random variables completed under the norm $\hat{\mathbb{E}}\left[|\cdot|^{p}\right]^{1 / p}$. Under this $G$-expectation framework ( $G$-framework for short) a new type of Brownian motion called $G$-Brownian motion was constructed. The stochastic calculus with respect to the $G$-Brownian motion has been established. The existence and uniqueness of solution of a SDE driven by $G$-Brownian motion can be proved in a way parallel to that in the classical SDE theory. But the solvability of BSDE driven by $G$-Brownian motion becomes a challenging problem. For a recent account and development of $G$-expectation

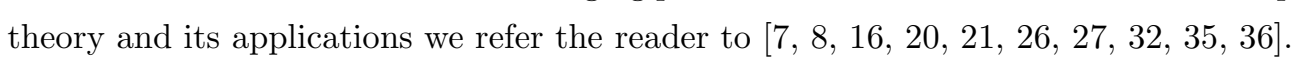

Let us mention that there are other recent advances and their applications in stochastic calculus that do not require a probability space framework. Denis and Martini [3] developed quasi-sure stochastic analysis, but they did not have conditional expectation. This topic was further examined by Denis et al. [4] and Soner et al. 33]. It is worthing to point out that Soner et al. 34] have obtained a deep result of existence and uniqueness theorem for a new type of fully nonlinear BSDE, called 2BSDE. Various stochastic control (game) problems are investigated in [13, 17, 18, 30] and the applications in finance are studied in [14, 15].

Recently $\mathrm{Hu}$ et. al studied the following BSDE driven by $G$-Brownian motion in [11] and [10]:

$$
\begin{aligned}
Y_{t} & =\xi+\int_{t}^{T} f\left(s, Y_{s}, Z_{s}\right) d s+\int_{t}^{T} g\left(s, Y_{s}, Z_{s}\right) d\langle B\rangle_{s} \\
& -\int_{t}^{T} Z_{s} d B_{s}-\left(K_{T}-K_{t}\right) .
\end{aligned}
$$

They proved that there exists a unique triple of processes $(Y, Z, K)$ within our $G$-framework which solves the above BSDE under a standard Lipschitz conditions on $f(s, y, z)$ and $g(s, y, z)$ in $(y, z)$. The decreasing $G$-martingale $\mathrm{K}$ is aggregated and the solution is time consistent. Some important properties of the BSDE driven by $G$-Brownian motion such as comparison theorem and Girsanov transformation were given in [10].

In this paper, we study a stochastic recursive optimal control problem in which the objective functional is described by the solution of a BSDE driven by $G$-Brownian motion. In more details, the state equation is governed by the following controlled SDE driven by $G$-Brownian motion

$$
\begin{aligned}
d X_{s}^{t, x, u} & =b\left(s, X_{s}^{t, x, u}, u_{s}\right) d s+h_{i j}\left(s, X_{s}^{t, x, u}, u_{s}\right) d\left\langle B^{i}, B^{j}\right\rangle_{s}+\sigma\left(s, X_{s}^{t, x, u}, u_{s}\right) d B_{s}, \\
X_{t}^{t, x, u} & =x .
\end{aligned}
$$

The objective functional is introduced by the solution $Y_{t}^{t, x, u}$ of the following BSDE driven by $G$-Brownian motion at time $t$ :

$$
\begin{aligned}
& -d Y_{s}^{t, x, u}=f\left(s, X_{s}^{t, x, u}, Y_{s}^{t, x, u}, Z_{s}^{t, x, u}, u_{s}\right) d s+g_{i j}\left(s, X_{s}^{t, x, u}, Y_{s}^{t, x, u}, Z_{s}^{t, x, u}, u_{s}\right) d\left\langle B^{i}, B^{j}\right\rangle_{s}-Z_{s}^{t, x, u} d B_{s}-d K_{s}^{t, x, u}, \\
& Y_{T}^{t, x, u}=\Phi\left(X_{T}^{t, x, u}\right), \quad s \in[t, T] .
\end{aligned}
$$


We define the value function of our stochastic recursive optimal control problem as follows:

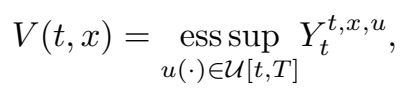

where the control set is in the $G$-framework.

It is well known that dynamic programming and related HJB equations is a powerful approach to solving optimal control problems (see [9], 37] and 28]). The objective of our paper is to establish the dynamic programming principle and investigate the value function in $G$-framework. The main result of this paper states that $V$ is deterministic continuous viscosity solution of the following HJB equation

$$
\begin{aligned}
& \partial_{t} V(t, x)+\sup _{u \in U} H\left(t, x, V, \partial_{x} V, \partial_{x x}^{2} V, u\right)=0, \\
& V(T, x)=\Phi(x), \quad x \in \mathbb{R}^{n},
\end{aligned}
$$

where

$$
\begin{aligned}
H(t, x, v, p, A, u) & =G(F(t, x, v, p, A, u))+\langle p, b(t, x, u)\rangle+f(t, x, v, \sigma(x, u) p, u), \\
F_{i j}(t, x, v, p, A, u) & =\left\langle A \sigma_{i}(t, x, u), \sigma_{j}(t, x, u)\right\rangle+2\left\langle p, h_{i j}(t, x, u)\right\rangle+2 g_{i j}(t, x, v, \sigma(x, u) p, u),
\end{aligned}
$$

$(t, x, v, p, A, u) \in[0, T] \times \mathbb{R}^{n} \times \mathbb{R} \times \mathbb{R}^{d} \times \mathbb{S}_{n} \times U$ and $\sigma_{i}$ is the $i$-th column of $\sigma$.

Notice that under the $G$-framework, there is no reference probability measure. Thus our results generalizes the results in Peng [28] and 29] which was only considered in the Wiener space (corresponding to $G$ is linear in our paper). Under a family of non-dominated probability measures, it is far from being trivial to prove that the value function $V$ is wellposed and deterministic. Furthermore, the BSDE driven by $G$-Brownian motion contains the decreasing $G$-martingale $K$, which is more difficult to deal with.

The paper is organized as follows. In section 2, we present some fundamental results on $G$-expectation theory and formulate our stochastic recursive optimal control problem. We establish the dynamic programming principle in section 3 . In section 4 , we first derive the HJB equation and prove that the value function is the viscosity solution of the obtained HJB equation.

\section{Preliminaries}

We review some basic notions and results of $G$-expectation, the related spaces of random variables and the backward stochastic differential equations driven by a $G$-Browninan motion. The readers may refer to [11, 22 25] for more details.

Let $\Omega$ be a given set and let $\mathcal{H}$ be a vector lattice of real valued functions defined on $\Omega$, namely $c \in \mathcal{H}$ for each constant $c$ and $|X| \in \mathcal{H}$ if $X \in \mathcal{H}$. $\mathcal{H}$ is considered as the space of random variables.

Definition 2.1 A sublinear expectation $\hat{\mathbb{E}}: \mathcal{H} \rightarrow \mathbb{R}$ satisfying the following properties: for all $X, Y \in \mathcal{H}$,

(i) Monotonicity: If $X \geq Y$ then $\hat{\mathbb{E}}[X] \geq \hat{\mathbb{E}}[Y]$;

(ii) Constant preservation: $\hat{\mathbb{E}}[c]=c$;

(iii) Sub-additivity: $\hat{\mathbb{E}}[X+Y] \leq \hat{\mathbb{E}}[X]+\hat{\mathbb{E}}[Y]$;

(iv) Positive homogeneity: $\hat{\mathbb{E}}[\lambda X]=\lambda \hat{\mathbb{E}}[X]$ for each $\lambda \geq 0$.

$(\Omega, \mathcal{H}, \hat{\mathbb{E}})$ is called a sublinear expectation space. 
Let $X_{1}$ and $X_{2}$ be two $n$-dimensional random vectors defined in sublinear expectation spaces $\left(\Omega_{1}, \mathcal{H}_{1}, \hat{\mathbb{E}}_{1}\right)$ and $\left(\Omega_{2}, \mathcal{H}_{2}, \hat{\mathbb{E}}_{2}\right)$ respectively. We will denote by $C_{\text {l.Lip }}\left(\mathbb{R}^{n}\right)$ the space of real continuous functions defined on $\mathbb{R}^{n}$ such that

$$
|\varphi(x)-\varphi(y)| \leq C\left(1+|x|^{k}+|y|^{k}\right)|x-y| \text { for all } x, y \in \mathbb{R}^{n},
$$

where $k$ and $C$ depend only on $\varphi$.

Definition 2.2 We call $X_{1}$ and $X_{2}$ identically distributed, denoted by $X_{1} \stackrel{d}{=} X_{2}$,

if for all $\varphi \in C_{l . L i p}\left(\mathbb{R}^{n}\right)$,

$$
\hat{\mathbb{E}}_{1}\left[\varphi\left(X_{1}\right)\right]=\hat{\mathbb{E}}_{2}\left[\varphi\left(X_{2}\right)\right]
$$

Definition 2.3 For given $(\Omega, \mathcal{H}, \hat{\mathbb{E}})$, random vectors $Y=\left(Y_{1}, \cdots, Y_{n}\right)$ and $X=\left(X_{1}, \cdots, X_{m}\right), Y_{i}, X_{i} \in \mathcal{H}$. We call $Y$ is independent of $X$ under $\hat{\mathbb{E}}[\cdot]$, denoted by $Y \perp X$, if for every test function $\varphi \in C_{\text {l.Lip }}\left(\mathbb{R}^{m} \times \mathbb{R}^{n}\right)$ we have

$$
\hat{\mathbb{E}}[\varphi(X, Y)]=\hat{\mathbb{E}}\left[\hat{\mathbb{E}}[\varphi(x, Y)]_{x=X}\right]
$$

Definition 2.4 (G-normal distribution) For given $(\Omega, \mathcal{H}, \hat{\mathbb{E}})$ and $X=\left(X_{1}, \cdots, X_{d}\right)$. $X$ is called $G$-normally distributed if for each $a, b \geq 0$, we have

$$
a X+b \bar{X} \stackrel{d}{=} \sqrt{a^{2}+b^{2}} X
$$

where $\bar{X}$ is an independent copy of $X$, i.e., $\bar{X} \stackrel{d}{=} X$ and $\bar{X} \perp X$.

For each $\varphi \in C_{l . L i p}\left(\mathbb{R}^{d}\right)$, we define

$$
u(t, x):=\hat{\mathbb{E}}[\varphi(x+\sqrt{t} X)], \quad(t, x) \in[0, \infty) \times \mathbb{R}^{d} .
$$

Peng [25] proved that $X$ is $G$-normally distributed if and only if $u$ is the solution of the following $G$-heat equation:

$$
\partial_{t} u-G\left(D_{x x}^{2} u\right)=0, u(0, x)=\varphi(x)
$$

where $G$ denotes the function

$$
G(A):=\frac{1}{2} \hat{\mathbb{E}}[\langle A X, X\rangle]: \mathbb{S}_{d} \rightarrow \mathbb{R}
$$

The function $G(\cdot): \mathbb{S}_{d} \rightarrow \mathbb{R}$ is a monotonic, sublinear mapping on $\mathbb{S}_{d}$, where $\mathbb{S}_{d}$ denotes the collection of $d \times d$ symmetric matrices. There exists a bounded and closed subset $\Gamma \subset \mathbb{R}^{d \times d}$ such that

$$
G(A)=\frac{1}{2} \sup _{\gamma \in \Gamma} \operatorname{tr}\left[\gamma \gamma^{T} A\right]
$$

where $\mathbb{R}^{d \times d}$ denotes the collection of $d \times d$ matrices.

In this paper we only consider non-degenerate $G$-normal distribution, i.e., there exists some $\underline{\sigma}^{2}>0$ such that $G(A)-G(B) \geq \underline{\sigma}^{2} \operatorname{tr}[A-B]$ for any $A \geq B$.

Let $\Omega=C_{0}\left([0, \infty) ; \mathbb{R}^{d}\right)$ be the space of real valued continuous functions on $[0, \infty)$ with $\omega_{0}=0$ and let $B_{t}(\omega)=\omega_{t}$ be the canonical process. Set

$$
L_{i p}(\Omega):=\left\{\varphi\left(B_{t_{1}}, \cdots, B_{t_{n}}\right): n \geq 1, t_{1}, \cdots, t_{n} \in[0, \infty), \varphi \in C_{l . L i p}\left(\mathbb{R}^{d \times n}\right)\right\}
$$


Let $\left\{\xi_{n}: n \geq 1\right\}$ be a sequence of identically distributed $d$-dimensional $G$-normally distributed random vectors in a sublinear expectation space $(\tilde{\Omega}, \tilde{\mathcal{H}}, \tilde{\mathbb{E}})$ such that $\xi_{i+1}$ is independent of $\left(\xi_{1}, \cdots, \xi_{i}\right)$ for every $i \geq 1$.

Definition 2.5 For each $X=\varphi\left(B_{t_{1}}-B_{t_{0}}, B_{t_{2}}-B_{t_{1}}, \cdots, B_{t_{m}}-B_{t_{m-1}}\right) \in L_{i p}(\Omega)$ with $0 \leq t_{0}<\cdots<t_{m}$, the $G$-expectation of $X$ is defined by

$$
\hat{\mathbb{E}}[X]=\tilde{\mathbb{E}}\left[\varphi\left(\sqrt{t_{1}-t_{0}} \xi_{1}, \cdots, \sqrt{t_{m}-t_{m-1}} \xi_{m}\right)\right] .
$$

The conditional G-expectation $\hat{\mathbb{E}}_{t}$ of $X$ with $t=t_{i}$ is defined by

$$
\begin{gathered}
\hat{\mathbb{E}}_{t_{i}}\left[\varphi\left(B_{t_{1}}-B_{t_{0}}, B_{t_{2}}-B_{t_{1}}, \cdots, B_{t_{m}}-B_{t_{m-1}}\right)\right] \\
=\tilde{\varphi}\left(B_{t_{1}}-B_{t_{0}}, B_{t_{2}}-B_{t_{1}}, \cdots, B_{t_{i}}-B_{t_{i-1}}\right)
\end{gathered}
$$

where

$$
\tilde{\varphi}\left(x_{1}, \cdots, x_{i}\right)=\hat{\mathbb{E}}\left[\varphi\left(x_{1}, \cdots, x_{i}, B_{t_{i+1}}-B_{t_{i}}, \cdots, B_{t_{m}}-B_{t_{m-1}}\right)\right] .
$$

$\left(\Omega, L_{i p}(\Omega), \hat{\mathbb{E}}\right)$ is called a $G$-expectation space. The corresponding canonical process $\left(B_{t}\right)_{t \geq 0}$ is called a $G$ Brownian motion.

We denote by $L_{G}^{p}(\Omega)$ the completion of $L_{i p}(\Omega)$ under the norm $\|X\|_{p, G}=\left(\hat{\mathbb{E}}\left[|X|^{p}\right]\right)^{1 / p}$ for $p \geq 1$. For each $t \geq 0, \hat{\mathbb{E}}_{t}[\cdot]$ can be extended continuously to $L_{G}^{1}(\Omega)$ under the norm $\|\cdot\|_{1, G}$. For each fixed $T>0$, set

$$
L_{i p}\left(\Omega_{T}\right):=\left\{\varphi\left(B_{t_{1}}, \cdots, B_{t_{n}}\right): n \geq 1, t_{1}, \cdots, t_{n} \in[0, T], \varphi \in C_{l . L i p}\left(\mathbb{R}^{d \times n}\right)\right\} .
$$

Obviously, $L_{i p}\left(\Omega_{T}\right) \subset L_{i p}(\Omega)$, then we can similarly define $L_{G}^{p}\left(\Omega_{T}\right)$ for $p \geq 1$.

Definition 2.6 Let $M_{G}^{0}(0, T)$ be the collection of processes in the following form: for a given partition $\left\{t_{0}, \cdots, t_{N}\right\}=\pi_{T}$ of $[0, T]$,

$$
\eta_{t}(\omega)=\sum_{j=0}^{N-1} \xi_{j}(\omega) I_{\left[t_{j}, t_{j+1}\right)}(t)
$$

where $\xi_{i} \in L_{i p}\left(\Omega_{t_{i}}\right), i=0,1,2, \cdots, N-1$.

We denote by $M_{G}^{p}(0, T)$ the completion of $M_{G}^{0}(0, T)$ under the norm $\|\eta\|_{M_{G}^{p}}=\left\{\hat{\mathbb{E}}\left[\int_{0}^{T}\left|\eta_{s}\right|^{p} d s\right]\right\}^{1 / p}$ for $p \geq 1$.

Theorem $2.7([4,10])$ There exists a family of weakly compact probability measures $\mathcal{P}$ on $(\Omega, \mathcal{B}(\Omega))$ such that

$$
\hat{\mathbb{E}}[\xi]=\sup _{P \in \mathcal{P}} E_{P}[\xi] \text { for all } \xi \in L_{G}^{1}(\Omega)
$$

$\mathcal{P}$ is called a set that represents $\hat{\mathbb{E}}$.

Let $\left\{W_{t}\right\}$ be a classical $d$-dimensional Brownian motion on a probability space $\left(\Omega^{0}, \mathcal{F}^{0}, P^{0}\right)$ and let $F^{0}=$ $\left\{\mathcal{F}_{t}^{0}\right\}$ be the augmented filtration generated by $W$. Set

$$
\mathcal{P}_{M}:=\left\{P_{\theta}: P_{\theta}=P^{0} \circ\left(B_{t}^{\theta, 0}\right)^{-1}, B_{t}^{\theta, 0}=\int_{0}^{t} \theta_{s} d W_{s}, \theta \in L_{F^{0}}^{2}([0, T] ; \Gamma)\right\},
$$

where $L_{F^{0}}^{2}([0, T] ; \Gamma)$ is the collection of $F^{0}$-adapted square integrable measurable processes with values in $\Gamma$. Set $\mathcal{P}=\overline{\mathcal{P}_{M}}$ the closure of $\mathcal{P}_{M}$ under the topology of weak convergence, then $\mathcal{P}$ is weakly compact. [4] proved that $\mathcal{P}$ represents $\hat{\mathbb{E}}$ on $L_{G}^{1}\left(\Omega_{T}\right)$. 
Proposition 2.8 ([4]) Let $\left\{P_{n}: n \geq 1\right\} \subset \mathcal{P}$ converge weakly to $P$. Then for each $\xi \in L_{G}^{1}(\Omega)$, we have $E_{P_{n}}[\xi] \rightarrow E_{P}[\xi]$.

For this $\mathcal{P}$, we define capacity

$$
c(A):=\sup _{P \in \mathcal{P}} P(A), A \in \mathcal{B}(\Omega) .
$$

A set $A \in \mathcal{B}(\Omega)$ is polar if $c(A)=0$. A property holds "quasi-surely" (q.s. for short) if it holds outside a polar set. In the following, we do not distinguish two random variables $X$ and $Y$ if $X=Y$ q.s.. We set

$$
\mathbb{L}^{p}\left(\Omega_{T}\right):=\left\{X \in \mathcal{B}\left(\Omega_{T}\right): \sup _{P \in \mathcal{P}} E_{P}\left[|X|^{p}\right]<\infty\right\} \text { for } p \geq 1 .
$$

It is important to note that $L_{G}^{p}\left(\Omega_{T}\right) \subset \mathbb{L}^{p}\left(\Omega_{T}\right)$.

\subsection{Forward and backward SDEs driven by G-Brownian motion}

We first give the definition of admissible controls.

Definition 2.9 For each $t \in[0, T], u$ is said to be an admissible control on $[t, T]$, if it satisfies the following conditions:

(i) $u:[t, T] \times \Omega \rightarrow U$ where $U$ is a compact set of $\mathbb{R}^{m}$;

(ii) $u \in M_{G}^{2}\left(t, T ; \mathbb{R}^{m}\right)$.

The set of admissible controls on $[t, T]$ is denoted by $\mathcal{U}[t, T]$.

In the rest of this paper, we use Einstein summation convention.

Let $t \in[0, T], \varepsilon>0, \xi \in L_{G}^{2+\varepsilon}\left(\Omega_{t}\right)$ and $u \in \mathcal{U}[t, T]$. Consider the following forward and backward SDEs driven by $G$-Brownian motion:

$$
\begin{aligned}
d X_{s}^{t, \xi, u} & =b\left(s, X_{s}^{t, \xi, u}, u_{s}\right) d s+h_{i j}\left(s, X_{s}^{t, \xi, u}, u_{s}\right) d\left\langle B^{i}, B^{j}\right\rangle_{s}+\sigma\left(s, X_{s}^{t, \xi, u}, u_{s}\right) d B_{s}, \\
X_{t}^{t, \xi, u} & =\xi
\end{aligned}
$$

and

$$
\begin{aligned}
& -d Y_{s}^{t, \xi, u}=f\left(s, X_{s}^{t, \xi, u}, Y_{s}^{t, \xi, u}, Z_{s}^{t, \xi, u}, u_{s}\right) d s+g_{i j}\left(s, X_{s}^{t, \xi, u}, Y_{s}^{t, \xi, u}, Z_{s}^{t, \xi, u}, u_{s}\right) d\left\langle B^{i}, B^{j}\right\rangle_{s}-Z_{s}^{t, \xi, u} d B_{s}-d K_{s}^{t, \xi, u}, \\
& Y_{T}^{t, \xi, u}=\Phi\left(X_{T}^{t, \xi, u}\right), \quad s \in[t, T],
\end{aligned}
$$

where

$$
\begin{aligned}
& b:[t, T] \times \mathbb{R}^{n} \times U \rightarrow \mathbb{R}^{n} ; \\
& h_{i j}:[t, T] \times \mathbb{R}^{n} \times U \rightarrow \mathbb{R}^{n} ; \\
& \sigma:[t, T] \times \mathbb{R}^{n} \times U \rightarrow \mathbb{R}^{n \times d} ; \\
& f:[t, T] \times \mathbb{R}^{n} \times \mathbb{R} \times \mathbb{R}^{d} \times U \rightarrow \mathbb{R} ; \\
& g_{i j}:[t, T] \times \mathbb{R}^{n} \times \mathbb{R} \times \mathbb{R}^{d} \times U \rightarrow \mathbb{R} ; \\
& \Phi: \mathbb{R}^{n} \rightarrow \mathbb{R} .
\end{aligned}
$$


Denote

$$
\begin{aligned}
& S_{G}^{0}(0, T)=\left\{h\left(t, B_{t_{1} \wedge t}, \cdots, B_{t_{n} \wedge t}\right): t_{1}, \ldots, t_{n} \in[0, T], h \in C_{b, L i p}\left(\mathbb{R}^{n+1}\right)\right\} \\
& S_{G}^{2}(0, T)=\left\{\text { the completion of } S_{G}^{0}(0, T) \text { under the norm }\|\eta\|_{S_{G}^{2}}=\left\{\hat{\mathbb{E}}\left[\sup _{t \in[0, T]}\left|\eta_{t}\right|^{2}\right]\right\}^{\frac{1}{2}}\right\} .
\end{aligned}
$$

For given $t, u$ and $\xi,\left(X^{t, \xi, u}\right)$ and $\left(Y^{t, \xi, u}, Z^{t, \xi, u}, K^{t, \xi, u}\right)$ are called solutions of the above forward and backward SDEs respectively if $\left(X^{t, \xi, u}\right) \in M_{G}^{2}\left(t, T ; \mathbb{R}^{n}\right) ;\left(Y^{t, \xi, u}, Z^{t, \xi, u}\right) \in S_{G}^{2}(0, T) \times M_{G}^{2}(0, T) ; K^{t, \xi, u}$ is a decreasing $G$-martingale with $K_{t}^{t, \xi, u}=0, K_{T}^{t, \xi, u} \in L_{G}^{2}\left(\Omega_{T}\right)$; (2.2) and (2.3) are satisfied respectively.

Assume $b, h_{i j}, \sigma, f, g_{i j}, \Phi$ are deterministic functions and satisfying the following conditions:

Assumption 2.10 There exists a constant $c>0$ such that

$$
\begin{aligned}
& \left|b\left(s, x^{1}, u\right)-b\left(s, x^{2}, v\right)\right|+\left|h_{i j}\left(s, x^{1}, u\right)-h_{i j}\left(s, x^{2}, v\right)\right|+\left|\sigma\left(s, x^{1}, u\right)-\sigma\left(s, x^{2}, v\right)\right| \\
\leq & c\left(\left|x^{1}-x^{2}\right|+|u-v|\right), \forall\left(s, x^{1}, u\right),\left(s, x^{2}, v\right) \in[t, T] \times \mathbb{R}^{n} \times U
\end{aligned}
$$

and $b, h_{i j}, \sigma$ are continuous about $t$.

Assumption 2.11 There exists a constant $c>0$ such that

$$
\begin{aligned}
& \left|f\left(s, x^{1}, y^{1}, z^{1}, u\right)-f\left(s, x^{2}, y^{2}, z^{2}, v\right)\right| \leq c\left(\left|x^{1}-x^{2}\right|+\left|y^{1}-y^{2}\right|+\left|z^{1}-z^{2}\right|+|u-v|\right) ; \\
& \left|g_{i j}\left(s, x^{1}, y^{1}, z^{1}, u\right)-g_{i j}\left(s, x^{2}, y^{2}, z^{2}, v\right)\right| \leq c\left(\left|x^{1}-x^{2}\right|+\left|y^{1}-y^{2}\right|+\left|z^{1}-z^{2}\right|+|u-v|\right) ; \\
& \left|\Phi\left(x^{1}\right)-\Phi\left(x^{2}\right)\right| \leq c\left|x^{1}-x^{2}\right|, \\
& \forall\left(s, x^{1}, y^{1}, z^{1}, u\right),\left(s, x^{2}, y^{2}, z^{2}, v\right) \in[t, T] \times \mathbb{R}^{n} \times \mathbb{R} \times \mathbb{R}^{d} \times U
\end{aligned}
$$

and $f, g_{i j}$ are continuous about $t$.

Remark 2.12 Suppose Assumptions (2.10) and (2.11) hold. Then there exists a constant $K>0$ such that

$$
\begin{aligned}
& |b(s, x, u)|+\left|h_{i j}(s, x, u)\right|+|\sigma(s, x, u)| \leq K(1+|x|) \\
& |f(s, x, 0,0, u)|+\left|g_{i j}(s, x, 0,0, u)\right| \leq c(1+|x|) \\
& |\Phi(x)| \leq K(1+|x|), \quad \forall(s, x, u) \in[t, T] \times \mathbb{R}^{n} \times U
\end{aligned}
$$

We have the following theorems.

Theorem 2.13 (25]) Let Assumption 2.10 hold. Then there exists a unique adapted solution $X$ for equation (2.2).

Theorem 2.14 ([10]) Let Assumption 2.11 hold. Then there exists a unique adapted solution $(Y, Z, K)$ for equation (2.3). 


\subsection{Stochastic optimal control problem}

The state equation of our stochastic optimal control problem is governed by the above forward SDE (2.2) and the objective functional is introduced by the solution of the BSDE (2.3) at time $t$. Let $\xi$ equals a constant $x \in \mathbb{R}^{n}$. When $u$ changes, $Y_{t}^{t, x, u}$ (the solution $Y^{t, x, u}$ at time $t$ ) also changes. In order to study the value function of our stochastic optimal control problem, we need to define the essential supremum of $\left\{Y_{t}^{t, x, u} \mid u \in \mathcal{U}[t, T]\right\}$.

Definition 2.15 The essential supremum of $\left\{Y_{t}^{t, x, u} \mid u \in \mathcal{U}[t, T]\right\}$, denoted by $\operatorname{esssup}_{u(\cdot) \in \mathcal{U}[t, T]} Y_{t}^{t, x, u}$, is a random variable $\zeta \in L_{G}^{2}\left(\Omega_{t}\right)$ satisfying:

(i). $\forall u \in \mathcal{U}[t, T], \zeta \geq Y_{t}^{t, x, u}$ q.s., and

(ii). if $\eta$ is a random variable satisfying $\eta \geq Y_{t}^{t, x, u}$ q.s. for any $u \in \mathcal{U}[t, T]$, then $\zeta \leq \eta$ q.s..

Remark 2.16 It is easy to verify that $c(A)=0$ if and only if $P(A)=0$ for each $P \in \mathcal{P}$. Thus $\zeta \leq \eta$ q.s. is equivalent to $\zeta \leq \eta P-$ a.s. for each $P \in \mathcal{P}$.

Proposition 2.17 Let $\zeta, \eta \in L_{G}^{2}(\Omega)$. If $\zeta \leq \eta P-$ a.s. for each $P \in \mathcal{P}_{M}$, then $\zeta \leq \eta$ q.s..

Proof. It is easy to check that $(\zeta-\eta)^{+} \in L_{G}^{2}(\Omega)$. By Proposition 2.8 we obtain

$$
\hat{\mathbb{E}}\left[(\zeta-\eta)^{+}\right]=\sup _{P \in \mathcal{P}} E_{P}\left[(\zeta-\eta)^{+}\right]=\sup _{P \in \mathcal{P}_{M}} E_{P}\left[(\zeta-\eta)^{+}\right]=0 .
$$

Thus $\zeta \leq \eta$ q.s..

Remark 2.18 From the above proposition, it is easy to deduce that $\zeta \leq \eta$ q.s. if and only if $\zeta \leq \eta P-$ a.s. for each $P \in \mathcal{P}_{M}$.

Our stochastic optimal control problem is: for given $x \in \mathbb{R}^{n}$, to find $u(\cdot) \in \mathcal{U}[t, T]$ so as to maximize the objective function $Y_{t}^{t, x, u}$.

The value function $V$ is defined to be

$$
V(t, x):=\operatorname{esssup}_{u(\cdot) \in \mathcal{U}[t, T]} Y_{t}^{t, x, u} .
$$

Next we prove that $V(t, x)$ exists and is deterministic, and then we show that it satisfies a kind of HJB equation.

For $x \in R^{n}, u(\cdot) \in \mathcal{U}[t, T]$ and $P \in \mathcal{P}_{M}$, we consider the following forward and backward equation:

$$
\begin{aligned}
& d X_{s}^{t, x, u ; P}=b\left(s, X_{s}^{t, x, u ; P}, u_{s}\right) d s+h_{i j}\left(s, X_{s}^{t, x, u ; P}, u_{s}\right) d\left\langle B^{i}, B^{j}\right\rangle_{s}+\sigma\left(s, X_{s}^{t, x, u ; P}, u_{s}\right) d B_{s}, \\
& X_{t}^{t, x, u ; P}=x, \quad P-a . s .
\end{aligned}
$$

and

$$
\begin{aligned}
& d Y_{s}^{t, x, u ; P}=-f\left(s, X_{s}^{t, x, u ; P}, Y_{s}^{t, x, u ; P}, Z_{s}^{t, x, u ; P}, u_{s}\right) d s-g_{i j}\left(s, X_{s}^{t, x, u ; P}, Y_{s}^{t, x, u ; P}, Z_{s}^{t, x, u ; P}, u_{s}\right) d\left\langle B^{i}, B^{j}\right\rangle_{s}+Z_{s}^{t, x, u ; P} d B_{s}, \\
& Y_{T}^{t, x, u ; P}=\Phi\left(X_{T}^{t, x, u ; P}\right), \quad s \in[t, T], \quad P-a . s . .
\end{aligned}
$$


Remark 2.19 Note that under probability $P \in \mathcal{P}_{M}$, the process $\left\{B_{s}\right\}_{t \leq s \leq T}$ in the equation (2.5) and (2.6) is generally not a standard Brownian Motion. But the martingale representation property still holds for $P$ (see [32] and [34]), thus there still exist unique solutions for (2.5) and (2.6).

By [25], we have

$$
X_{s}^{t, x, u ; P}=X_{s}^{t, x, u} \quad P-a . s . .
$$

Soner et al. 34] give the following representation for the solution $Y^{t, x, u}$ of (2.3):

$$
Y_{t}^{t, x, u}=\operatorname{esssup}_{Q \in \mathcal{P}_{M}(t, P)} P Y_{t}^{t, x, u ; Q}, \quad P-a . s .,
$$

where $\operatorname{esssup}^{P}$ is the esssup with respect to probability $P$ in the classical sense and

$$
\mathcal{P}_{M}(t, P):=\left\{Q: Q(A)=P(A), \forall A \in \mathcal{F}_{t}, Q \in \mathcal{P}_{M}\right\} .
$$

For each fix $P \in \mathcal{P}_{M}$, the value function $V^{P}$ is defined to be

$$
V^{P}(t, x):=\operatorname{esssup}_{u(\cdot) \in \mathcal{U}[t, T]} \operatorname{esssup}_{Q \in \mathcal{P}_{M}(t, P)} P Y_{t}^{t, x, u ; Q}, \quad P-a . s . .
$$

Remark 2.20 If $V^{P}(t, x)$ is a deterministic function and independent of $P$, then by Remark 2.18, we have $V(t, x)=V^{P}(t, x)$.

\section{Dynamic programming principle}

For given initial data $(t, x)$, a positive real number $\delta \leq T-t$ and $\eta \in L_{G}^{2}\left(\Omega_{t+\delta}\right)$, we define

$$
\mathbb{G}_{t, t+\delta}^{t, x, u}[\eta]:=Y_{t}^{t, x, u},
$$

where $\left(X_{s}^{t, x, u}, Y_{s}^{t, x, u}, Z_{s}^{t, x, u}\right)_{t \leq s \leq t+\delta}$ is the solution of the following forward and backward equations:

$$
\begin{aligned}
d X_{s}^{t, x, u} & =b\left(s, X_{s}^{t, x, u}, u_{s}\right) d s+h_{i j}\left(s, X_{s}^{t, x, u}, u_{s}\right) d\left\langle B^{i}, B^{j}\right\rangle_{s}+\sigma\left(s, X_{s}^{t, x, u}, u_{s}\right) d B_{s}, \\
X_{t}^{t, x, u} & =x
\end{aligned}
$$

and

$$
\begin{aligned}
& -d Y_{s}^{t, x, u}=f\left(s, X_{s}^{t, x, u}, Y_{s}^{t, x, u}, Z_{s}^{t, x, u}, u_{s}\right) d s+g_{i j}\left(s, X_{s}^{t, x, u}, Y_{s}^{t, x, u}, Z_{s}^{t, x, u}, u_{s}\right) d\left\langle B^{i}, B^{j}\right\rangle_{s}-Z_{s}^{t, x, u} d B_{s}-d K_{s}^{t, x, u}, \\
& Y_{t+\delta}^{t, x, u}=\eta, \quad s \in[t, t+\delta] .
\end{aligned}
$$

Note that $\mathbb{G}_{t, t+\delta}^{t, x, u}[\cdot]$ is a (backward) semigroup which was first introduced by Peng in [29].

Now we give some notations:

$$
\begin{aligned}
& L_{i p}\left(\Omega_{s}^{t}\right):=\left\{\varphi\left(B_{t_{1}}-B_{t}, \ldots, B_{t_{n}}-B_{t}\right): n \geq 1, t_{1}, \ldots, t_{n} \in[t, s], \varphi \in C_{l . L i p}\left(\mathbb{R}^{d \times n}\right)\right\} ; \\
& M_{G}^{0, t}(t, T):=\left\{\eta_{s}=\sum_{j=0}^{N-1} \xi_{j} I_{\left[t_{j}, t_{j+1}\right)}(s): s \in[t, T], t=t_{0}<\cdots<t_{N}=T, \xi_{i} \in L_{i p}\left(\Omega_{t_{i}}^{t}\right)\right\} ; \\
& M_{G}^{2, t}(t, T):=\left\{\text { the completion of } M_{G}^{0, t}(t, T) \text { under }\|\cdot\|_{M_{G}^{2}}\right\} ; \\
& \mathcal{U}^{t}[t, T]:=\left\{u \in M_{G}^{2, t}\left(t, T ; \mathbb{R}^{m}\right) \text { with values in } U\right\} ; \\
& \mathcal{U}_{0}[t, T]:=\left\{u=\sum_{i=1}^{m} 1_{A_{i}} u^{i}: m \in \mathbb{N}, u^{i} \in \mathcal{U}^{t}[t, T], \text { where }\left\{A_{i}\right\}_{i=1, \ldots m} \text { is a partition of } \Omega, A_{i} \in \mathcal{B}\left(\Omega_{s}\right)\right\} .
\end{aligned}
$$


Our main result in this section is the following dynamic programming principle.

Theorem 3.1 Let Assumptions 2.10 and 2.11 hold. Then for any $t \leq T, x \in \mathbb{R}^{n}, V(t, x)$ exists and is deterministic. Furthermore, for any $s \in[t, T]$, we have

$$
\begin{aligned}
V(t, x) & =\operatorname{essup}_{u(\cdot) \in \mathcal{U}[t, s]} \mathbb{G}_{t, s}^{t, x, u}\left[V\left(s, X_{s}^{t, x, u}\right)\right] \\
& =\sup _{u(\cdot) \in \mathcal{U}^{t}[t, s]} \mathbb{G}_{t, s}^{t, x, u}\left[V\left(s, X_{s}^{t, x, u}\right)\right] .
\end{aligned}
$$

In order to prove Theorem 3.1, we need to study $V^{P}(t, x)$ through equations (2.5) and (2.6). The following priori estimates are classical and we omit the proof (refer to [6]).

Lemma 3.2 Under Assumptions 2.10] and [2.11, for any $\xi_{1}, \xi_{2} \in L_{G}^{2}\left(\Omega_{t}\right)$ and $u, v \in \mathcal{U}[t, T]$, there exists a constant $C_{2}$ such that

$$
\begin{aligned}
& E_{P}\left[\sup _{s \in[t, T]}\left|X_{s}^{t, \xi_{1}, u ; P}-X_{s}^{t, \xi_{2}, u ; P}\right|^{2} \mid \mathcal{F}_{t}\right] \leq C_{2}\left|\xi_{1}-\xi_{2}\right|^{2} ; \\
& \left|Y_{t}^{t, \xi_{1}, u ; P}-Y_{t}^{t, \xi_{2}, u ; P}\right|^{2} \leq C_{2}\left|\xi_{1}-\xi_{2}\right|^{2} ; \\
& \left|Y_{t}^{t, \xi_{1}, u ; P}-Y_{t}^{t, \xi_{1}, v ; P}\right|^{2} \leq C_{2} E_{P}\left[\int_{t}^{T}\left|u_{s}-v_{s}\right|^{2} d s \mid \mathcal{F}_{t}\right], \quad P-\text { a.s.. }
\end{aligned}
$$

The following theorem shows that $V^{P}(t, x)$ is deterministic and independent of $P \in \mathcal{P}_{M}$.

Theorem 3.3 Under Assumptions (2.10) and (2.11), we have

(i) For a fixed $P \in \mathcal{P}_{M}, V^{P}(t, x)$ is a deterministic function and

$$
V^{P}(t, x)=\underset{u \in \mathcal{U}^{t}[t, T] Q \in \mathcal{P}_{M}(t, P)}{\operatorname{ess} \sup _{t}} \operatorname{essup}^{P} Y_{t}^{t, x, u ; Q}, P-a . s .
$$

(ii) For each $u \in \mathcal{U}^{t}[t, T], Y_{t}^{t, x, u}$ (the solution of (3.1) at time $t$ ) is a deterministic function. Furthermore,

$$
V(t, x)=\sup _{u \in \mathcal{U}^{t}[t, T]} Y_{t}^{t, x, u}
$$

Proof: (i) Without loss of generality, for (2.5) and (2.6), we only study the case $n=d=1$ and $h_{i j}=g_{i j}=0$. By the definition of $V^{P}(t, x)$,

$$
V^{P}(t, x) \geq \operatorname{esssup}_{u \in \mathcal{U}^{t}[t, T]}{\operatorname{ess} \sup _{Q \in \mathcal{P}_{M}(t, P)} P}_{Y_{t}^{t, x, u ; Q}} .
$$

Analysis similar to that in Lemma 43 in [4] shows that $\mathcal{U}_{0}[t, T]$ is dense in $\mathcal{U}[t, T]$ under probability $Q$. It yields that

$$
\begin{aligned}
& V^{P}(t, x)=\operatorname{essip}_{u(\cdot) \in \mathcal{U}[t, T]} \operatorname{ess~sup}_{Q \in \mathcal{P}_{M}(t, P)} P Y_{t}^{t, x, u ; Q} \\
& =\operatorname{esssup}_{Q \in \mathcal{P}_{M}(t, P)} P \operatorname{esssup}_{u(\cdot) \in \mathcal{U}[t, T]} Y_{t}^{t, x, u ; Q} \\
& =\operatorname{essips}_{Q \in \mathcal{P}_{M}(t, P)} P \operatorname{esssup}_{u \in \mathcal{U}_{0}[t, T]} Y_{t}^{t, x, u ; Q} \\
& =\operatorname{esssup}_{u \in \mathcal{U}_{0}[t, T]} \operatorname{esssup}_{Q \in \mathcal{P}_{M}(t, P)} P Y_{t}^{t, x, u ; Q} .
\end{aligned}
$$


Set $u=\sum_{i=1}^{m} 1_{A_{i}} u^{i} \in \mathcal{U}_{0}[t, T]$. Consider the following equation

$$
\begin{aligned}
& X_{s}^{t, x, u^{i} ; Q}=x+\int_{t}^{s} b\left(r, X_{r}^{t, x, u^{i} ; Q}, u_{r}^{i}\right) d r+\int_{t}^{s} \sigma\left(r, X_{r}^{t, x, u^{i} ; Q}, u_{r}^{i}\right) d B_{r}, \\
& Y_{s}^{t, x, u^{i} ; Q}=\Phi\left(X_{T}^{t, x, u^{i} ; Q}\right)+\int_{s}^{T} f\left(r, X_{r}^{t, x, u^{i} ; Q}, Y_{r}^{t, x, u^{i} ; Q}, Z_{r}^{t, x, u^{i} ; Q}, u_{r}^{i}\right) d r-\int_{s}^{T} Z_{r}^{t, x, u^{i} ; Q} d B_{r} .
\end{aligned}
$$

Multiplying by $I_{A_{i}}$ and adding the corresponding terms, we obtain

$$
\begin{aligned}
\sum_{i=1}^{N} 1_{A_{i}} X_{s}^{t, x, u^{i} ; Q}= & x+\sum_{i=1}^{N} 1_{A_{i}} \int_{t}^{s} b\left(r, X_{r}^{t, x, u^{i} ; Q}, u_{r}^{i}\right) d r+\sum_{i=1}^{N} 1_{A_{i}} \int_{t}^{s} \sigma\left(r, X_{r}^{t, x, u^{i} ; Q}, u_{r}^{i}\right) d B_{r}, \\
\sum_{i=1}^{N} 1_{A_{i}} Y_{s}^{t, x, u^{i} ; Q}= & \sum_{i=1}^{N} 1_{A_{i}} \Phi\left(X_{T}^{t, x, u^{i} ; Q}\right)-\sum_{i=1}^{N} 1_{A_{i}} \int_{s}^{T} Z_{r}^{t, x, u^{i} ; Q} d B_{r} \\
& +\sum_{i=1}^{N} 1_{A_{i}} \int_{s}^{T} f\left(r, X_{r}^{t, x, u^{i} ; Q}, Y_{r}^{t, x, u^{i} ; Q}, Z_{r}^{t, x, u^{i} ; Q}, u_{r}^{i}\right) d r .
\end{aligned}
$$

Then

$$
\begin{aligned}
\sum_{j=1}^{N} 1_{A_{i}} X_{s}^{t, x, u^{i} ; Q}= & x+\int_{t}^{s} b\left(r, \sum_{j=1}^{N} 1_{A_{i}} X_{r}^{t, x, u^{i} ; Q}, \sum_{j=1}^{N} 1_{A_{i}} u_{r}^{i}\right) d r+\int_{t}^{s} \sigma\left(r, \sum_{j=1}^{N} 1_{A_{i}} X_{r}^{t, x, u^{i} ; Q}, \sum_{j=1}^{N} 1_{A_{i}} u_{r}^{i}\right) d B_{r}, \\
\sum_{j=1}^{N} 1_{A_{i}} Y_{s}^{t, x, u^{i} ; Q}= & \Phi\left(\sum_{j=1}^{N} 1_{A_{i}} X_{T}^{t, x, u^{i} ; Q}\right)-\int_{s}^{T}\left(\sum_{j=1}^{N} 1_{A_{i}} Z_{r}^{t, x, u^{i} ; Q}\right) d B_{r} \\
& +\int_{s}^{T} f\left(r, \sum_{i=1}^{N} 1_{A_{i}} X_{r}^{t, x, u^{i} ; Q}, \sum_{i=1}^{N} 1_{A_{i}} Y_{r}^{t, x, u^{i} ; Q}, \sum_{i=1}^{N} 1_{A_{i}} Z_{r}^{t, x, u^{i} ; Q}, \sum_{i=1}^{N} 1_{A_{i}} u_{r}^{i}\right) d r .
\end{aligned}
$$

By the uniqueness theorem of BSDE, we have

$$
Y_{t}^{t, x, u ; Q}=\sum_{i=1}^{N} 1_{A_{i}} Y_{t}^{t, x, u^{i} ; Q} \leq \sum_{i=1}^{N} 1_{A_{i}} \operatorname{ess}_{u \in \mathcal{U}^{t}[t, T]} \operatorname{ess~sup}_{Q \in \mathcal{P}_{M}(t, P)} P Y_{t}^{t, x, u ; Q} .
$$

From this we get

$$
V^{P}(t, x) \leq \operatorname{ess}_{u \in \mathcal{U}^{t}[t, T] Q \in \mathcal{P}_{M}(t, P)} \operatorname{esssup}^{P} Y_{t}^{t, x, u ; Q}, P-a . s . .
$$

Thus

$$
V^{P}(t, x)=\underset{u \in \mathcal{U}^{t}[t, T] Q \in \mathcal{P}_{M}(t, P)}{\operatorname{ess} \sup } \operatorname{essup}_{t} P Y_{t}^{t, x, u ; Q} .
$$

(ii) For each $u \in \mathcal{U}^{t}[t, T]$, it is easy to check that $Y_{t}^{t, x, u}$ is a deterministic function. Note that

$$
Y_{t}^{t, x, u}=\operatorname{essiup}_{Q \in \mathcal{P}_{M}(t, P)}{ }^{P} Y_{t}^{t, x, u ; Q} P-\text { a.s.. }
$$

Thus for each $P \in \mathcal{P}_{M}$, we obtain

$$
V^{P}(t, x)=\sup _{u \in \mathcal{U}^{t}[t, T]} Y_{t}^{t, x, u} P-a . s .
$$

which implies that $V^{P}(t, x)$ is a constant and independent of $P$. By the definition of $V(t, x)$, we deduce that $V(t, x)$ is deterministic and

$$
V(t, x)=\sup _{u \in \mathcal{U}^{t}[t, T]} Y_{t}^{t, x, u}
$$

This completes the proof.

We have the following estimations of the continuity of value function $V(t, x)$ with respect to $x$. 
Lemma 3.4 $\forall t \in[0, T]$ and $x, x^{\prime} \in \mathbb{R}^{n}$, there exists a constant $C_{0}$ such that

(i) $\left|V(t, x)-V\left(t, x^{\prime}\right)\right| \leq C_{0}\left|x-x^{\prime}\right|$;

(ii) $|V(t, x)| \leq C_{0}(1+|x|)$.

Proof. By Lemma 3.2, we have

$$
\left|Y_{t}^{t, x, u ; Q}-Y_{t}^{t, x^{\prime}, u ; Q}\right| \leq C_{0}\left|x-x^{\prime}\right|, \quad P-\text { a.s.. }
$$

It is easy to verify that for any $P \in \mathcal{P}_{M}$,

$$
\begin{aligned}
\left|V(t, x)-V\left(t, x^{\prime}\right)\right| & \leq \sup _{u(\cdot) \in \mathcal{U}[t, T]} \operatorname{esssup}_{Q \in \mathcal{P}_{M}(t, P)} P\left|Y_{t}^{t, x, u ; Q}-Y_{t}^{t, x^{\prime}, u ; Q}\right| \\
& \leq C_{0}\left|x-x^{\prime}\right|
\end{aligned}
$$

This completes the proof.

$\forall s \geq t$, define

$$
\begin{aligned}
& \mathbb{M}^{2,0}(s, T)=\left\{\eta_{t}=\sum_{i=0}^{N-1} \xi_{t_{i}} I_{\left[t_{i}, t_{i+1}\right)}(t): s=t_{0}<\cdots<t_{N}=T, \xi_{t_{i}} \in \mathbb{L}^{2}\left(\Omega_{t_{i}}\right)\right\} ; \\
& \mathbb{M}_{G}^{2}(s, T)=\left\{\text { the completion of } \mathbb{M}^{2,0}(s, T) \text { under }\|\eta\|_{\mathbb{M}^{2}}:=\left(\hat{\mathbb{E}}\left[\int_{0}^{T}\left|\eta_{t}\right|^{2} d t\right]\right)^{1 / 2}\right\} ; \\
& \mathbb{U}[s, T]=\left\{u:[s, T] \times \Omega \rightarrow U: u \in \mathbb{M}_{G}^{2}\left(s, T ; \mathbb{R}^{m}\right)\right\} .
\end{aligned}
$$

Lemma 3.5 Suppose $s \in[0, T]$ and $\xi \in L_{G}^{2}\left(\Omega_{s}\right)$. Then we have

(i) for any $v(\cdot) \in \mathcal{U}[s, T]$ and $Q \in \mathcal{P}_{M}(s, P)$,

$$
V(s, \xi) \geq Y_{s}^{s, \xi, v ; Q}, \quad P-a . s .
$$

(ii) for any $\varepsilon>0$, there is an admissible control $v^{\prime}(\cdot) \in \mathbb{U}[s, T]$ and $Q^{\prime} \in \mathcal{P}_{M}(s, P)$ such that

$$
V(s, \xi) \leq Y_{s}^{s, \xi_{s}, v^{\prime} ; Q^{\prime}}+\varepsilon, \quad P-a . s .
$$

(iii)

$$
V(s, \xi)=\underset{v(\cdot) \in \mathcal{U}[s, T]}{\operatorname{esssup}} Y_{s}^{s, \xi, v} .
$$

Proof. (i) Set

$$
\xi=\sum_{i=1}^{N} 1_{A_{i}} x^{i} \in \mathbb{L}\left(\Omega_{s}\right),
$$

where $\left\{A_{i}\right\}_{i=1, \ldots N}$ is a partition of $\Omega, A_{i} \in \mathcal{B}\left(\Omega_{s}\right)$ and $x^{i} \in \mathbb{R}^{n}$.

For any $v(\cdot) \in \mathcal{U}[s, T], Q \in \mathcal{P}_{M}(s, P)$, we have

$$
Y_{s}^{s, \xi, v ; Q}=\sum_{i=1}^{N} 1_{A_{i}} Y_{s}^{s, x^{i}, v ; Q} \leq \sum_{i=1}^{N} 1_{A_{i}} V\left(s, x^{i}\right)=V\left(s, \sum_{i=1}^{N} 1_{A_{i}} x^{i}\right)=V(s, \xi) .
$$

For the general case, note that $V$ is continuous in $x$ and $Y_{s}^{s, \xi, v ; Q}$ is continuous in $\xi$. We can choose a sequence of simple random variables $\left\{\xi^{i}\right\}(i=1,2, \ldots)$ which converges to $\xi$. Using similar techniques in Lemma 3.2 and 3.4 we have 


$$
E_{P}\left|Y_{s}^{s, \xi, v ; Q}-Y_{s}^{s, \xi^{i}, v ; Q}\right|^{2} \rightarrow 0, \quad E_{P}\left|V(s, \xi)-V\left(s, \xi^{i}\right)\right|^{2} \rightarrow 0 .
$$

Then (3.5) holds.

(ii) For $\xi \in L_{G}^{2}\left(\Omega_{s}\right)$, we can construct a random variable

$$
\eta=\sum_{i=1}^{\infty} 1_{A_{i}} x^{i} \in \mathbb{L}^{2}\left(\Omega_{s}\right)
$$

such that

$$
|\eta-\xi| \leq \frac{\varepsilon}{3 C}
$$

where $C:=\max \left\{C_{0}, C_{2}\right\}$.

By Lemma 3.2 and 3.4 , for any $v(\cdot) \in \mathcal{U}[s, T]$,

$$
\left|Y_{s}^{s, \eta, v ; Q}-Y_{s}^{s, \xi, v ; Q}\right| \leq \frac{\varepsilon}{3},|V(s, \eta)-V(s, \xi)| \leq \frac{\varepsilon}{3} .
$$

For every $x^{i}$, we can choose an admissible control $v^{i}(\cdot) \in \mathcal{U}[s, T]$ and $Q^{i}$ such that

$$
V\left(s, x^{i}\right) \leq Y_{s}^{s, x^{i}, v^{i} ; Q^{i}}+\frac{\varepsilon}{3}, \quad P-a . s . .
$$

Denote

$$
\begin{aligned}
& v(\cdot):=\sum_{i=1}^{\infty} 1_{A_{i}} v^{i}(\cdot) \in \mathbb{U}[s, T], \\
& Q^{\prime}(A):=\sum_{i=1}^{\infty} Q^{i}\left(A \cap A_{i}\right), \quad \forall A \in \mathcal{B}\left(\Omega_{T}\right) .
\end{aligned}
$$

We have

$$
\begin{aligned}
& Y_{s}^{s, \xi, v ; Q^{\prime}} \geq-\left|Y_{s}^{s, \eta, v ; Q^{\prime}}-Y_{s}^{s, \xi, v ; Q^{\prime}}\right|+Y_{s}^{s, \eta, v ; Q^{\prime}} \\
& \geq-\frac{\varepsilon}{3}+\sum_{i=1}^{\infty} 1_{A_{i}} Y_{s}^{s, x^{i}, v^{i} ; Q^{i}} \\
& \geq-\frac{\varepsilon}{3}+\sum_{i=1}^{\infty} 1_{A_{i}}\left(V\left(s, x^{i}\right)-\frac{\varepsilon}{3}\right) \\
& =-\frac{2 \varepsilon}{3}+\sum_{i=1}^{\infty} 1_{A_{i}} V\left(s, x^{i}\right) \\
& =-\frac{2 \varepsilon}{3}+V(s, \eta) \\
& \geq-\varepsilon+V(s, \xi), \quad P-a . s .
\end{aligned}
$$

(iii) By (3.5) and (3.6), it is easy to prove (3.7).

The proof is completed.

Define the (backward) semigroup

$$
G_{t, s}^{t, x, u ; P}[\eta]=Y_{t}^{t, x, u ; P},
$$


where $\eta \in L_{G}^{2+\varepsilon}\left(\Omega_{s}\right)$ and $\left(X_{r}^{t, x, u ; P}, Y_{r}^{t, x, u ; P}, Z_{r}^{t, x, u ; P}\right)_{t \leq r \leq s}$ is the solution of the following forward-backward system:

$$
\begin{aligned}
d X_{s}^{t, x, u ; P} & =b\left(s, X_{s}^{t, x, u ; P}, u_{s}\right) d s+h_{i j}\left(s, X_{s}^{t, x, u ; P}, u_{s}\right) d\left\langle B^{i}, B^{j}\right\rangle_{s}+\sigma\left(s, X_{s}^{t, x, u ; P}, u_{s}\right) d B_{s}, \\
X_{t}^{t, x, u ; P} & =x
\end{aligned}
$$

and

$$
\begin{aligned}
-d Y_{r}^{t, x, u ; P}= & f\left(X_{r}^{t, x, u ; P}, Y_{r}^{t, x, u ; P}, Z_{r}^{t, x, u ; P}, u_{r}\right) d r-Z_{r}^{t, x, u ; P} d B_{r} \\
& +g_{i j}\left(X_{r}^{t, x, u ; P}, Y_{r}^{t, x, u ; P}, Z_{r}^{t, x, u ; P}, u_{r}\right) d\left\langle B^{i}, B^{j}\right\rangle_{r}, \\
Y_{s}^{t, x, u ; P}= & \eta, \quad r \in[t, s], \quad P-\text { a.s.. }
\end{aligned}
$$

It is obvious that for $Q \in \mathcal{P}_{M}(t, P)$

$$
G_{t, T}^{t, x, u ; Q}\left[\Phi\left(X_{T}^{t, x, u ; Q}\right)\right]=G_{t, s}^{t, x, u ; Q}\left[Y_{s}^{t, x, u ; Q}\right]
$$

Now we give the proof of Theorem 3.1

Proof. By Theorem 3.3 , for each fixed $P \in \mathcal{P}_{M}$, we have

$$
\begin{aligned}
V(t, x) & =\operatorname{esssup}_{u(\cdot) \in \mathcal{U}^{t}[t, T]} \operatorname{essips}_{Q \in \mathcal{P}_{M}(t, P)} P G_{t, T}^{t, x, u ; Q}\left[\Phi\left(X_{T}^{t, x, u ; Q}\right)\right] \\
& =\operatorname{ess~sup}_{u(\cdot) \in \mathcal{U}^{t}[t, s]} \operatorname{ess~sup}_{Q \in \mathcal{P}_{M}(t, P)} P G_{t, s}^{t, x, u ; Q}\left[Y_{s}^{t, x, u ; Q}\right] \\
& =\operatorname{essip}_{u(\cdot) \in \mathcal{U}[t, s]} \operatorname{ess~sup}_{Q \in \mathcal{P}_{M}(t, P)}{ }^{P} G_{t, s}^{t, x, u ; Q}\left[Y_{s}^{s, X_{s}^{t, x, u}, u ; Q}\right], \quad P-\text { a.s.. }
\end{aligned}
$$

By Lemma 3.5 and the comparison theorem of BSDE, we have

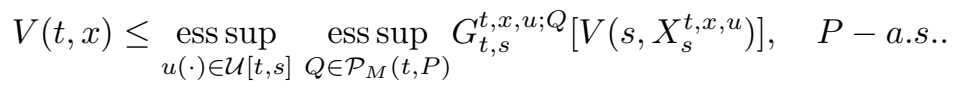

On the other hand, for each fixed $u(\cdot) \in \mathcal{U}[t, s], \forall \varepsilon>0$, by Lemma 3.5. there exist $\bar{u}(\cdot) \in \mathbb{U}[s, T]$ and $\tilde{Q} \in \mathcal{P}_{M}(s, Q)$ such that

$$
V\left(s, X_{s}^{t, x, u}\right) \leq Y_{s}^{s, X_{s}^{t, x, u}, \bar{u} ; \tilde{Q}}+\varepsilon, \quad Y_{t}^{t, x, \tilde{u} ; \tilde{Q}} \leq V(t, x),
$$

where

$$
\tilde{u}_{s}=1_{\{t \leq r \leq s\}} u_{r}+1_{\{s<r \leq T\}} \bar{u}_{r} .
$$

By the above inequality and the comparison theorem, we have

$$
\begin{aligned}
& Y_{s}^{s, X_{s}^{t, x, u}, \tilde{u} ; \tilde{Q}} \geq V\left(s, X_{s}^{t, x, u}\right)-\varepsilon \\
& V(t, x) \geq G_{t, s}^{t, x, u ; Q}\left[Y_{s}^{s, X_{s}^{t, x, u}, \tilde{u} ; \tilde{Q}}\right] \geq G_{t, s}^{t, x, u ; Q}\left[V\left(s, X_{s}^{t, x, u}\right)-\varepsilon\right] .
\end{aligned}
$$

By Lemma 3.2 there exists a constant $C_{0}$ such that 


$$
V(t, x) \geq G_{t, s}^{t, x, u ; Q}\left[V\left(s, X_{s}^{t, x, u}\right)\right]-C_{0} \varepsilon
$$

From this we get

$$
V(t, x) \geq \operatorname{esssup}_{u(\cdot) \in \mathcal{U}[t, s]} \operatorname{essip}_{Q \in \mathcal{P}_{M}(t, P)} P G_{t, s}^{t, x, u ; Q}\left[V\left(s, X_{s}^{t, x, u}\right)\right]-C_{0} \varepsilon .
$$

Thus by letting $\varepsilon \downarrow 0$, we obtain

$$
V(t, x)=\operatorname{esssup}_{u(\cdot) \in \mathcal{U}[t, s]} \operatorname{essipup}_{Q \in \mathcal{P}_{M}(t, P)} P G_{t, s}^{t, x, u ; Q}\left[V\left(s, X_{s}^{t, x, u ; Q}\right)\right], \quad P-a . s . .
$$

Similar to the proof of Theorem 3.3 , we can get

$$
V(t, x)=\operatorname{ess~sup}_{u(\cdot) \in \mathcal{U}^{t}[t, s]} \operatorname{esssup}_{Q \in \mathcal{P}_{M}(t, P)}{ }^{P} G_{t, s}^{t, x, u ; Q}\left[V\left(s, X_{s}^{t, x, u ; Q}\right)\right], \quad P-a . s . .
$$

Note that

$$
\begin{aligned}
& \operatorname{essiup}_{Q \in \mathcal{P}_{M}(t, P)}{ }^{P} G_{t, s}^{t, x, u ; Q}\left[V\left(s, X_{s}^{t, x, u}\right)\right] \\
= & \mathbb{G}_{t, s}^{t, x, u}\left[V\left(s, X_{s}^{t, x, u}\right)\right] P-\text { a.s. }
\end{aligned}
$$

We have

$$
\begin{aligned}
V(t, x) & =\operatorname{ess~sup}_{u(\cdot) \in \mathcal{U}[t, s]} \mathbb{G}_{t, s}^{t, x, u}\left[V\left(s, X_{s}^{t, x, u}\right)\right] \\
& =\sup _{u(\cdot) \in \mathcal{U}^{t}[t, s]} \mathbb{G}_{t, s}^{t, x, u}\left[V\left(s, X_{s}^{t, x, u}\right)\right] .
\end{aligned}
$$

This completes the proof.

The following lemma show the continuity of $V$ about $t$.

Lemma 3.6 The value function $V$ is $\frac{1}{2}$ Hölder continuous in $t$.

Proof. Set $(t, x) \in \mathbb{R}^{n} \times[0, T]$ and $\delta>0$. By dynamic programming principle, $\forall \varepsilon>0$, there exist $u(\cdot) \in$ $\mathcal{U}^{t}$ such that

$$
\mathbb{G}_{t, t+\delta}^{t, x, u}\left[V\left(t+\delta, X_{t+\delta}^{t, x, u}\right)\right]+\varepsilon \geq V(t, x) \geq \mathbb{G}_{t, t+\delta}^{t, x, u}\left[V\left(t+\delta, X_{t+\delta}^{t, x, u}\right)\right] .
$$

We first show that there exists $C>0$ such that $V(t+\delta, x)-V(t, x) \leq C \delta^{\frac{1}{2}}$. Similarly, we can prove $V(t+\delta, x)-V(t, x) \geq-C \delta^{\frac{1}{2}}$.

By equation (3.8), we have

$$
V(t+\delta, x)-V(t, x) \leq I_{\delta}^{1}+I_{\delta}^{2}
$$

where

$$
\begin{aligned}
I_{\delta}^{1} & =\mathbb{G}_{t, t+\delta}^{t, x, u}[V(t+\delta, x)]-\mathbb{G}_{t, t+\delta}^{t, x, u}\left[V\left(t+\delta, X_{t+\delta}^{t, x, u}\right)\right] \\
I_{\delta}^{2} & =V(t+\delta, x)-\mathbb{G}_{t, t+\delta}^{t, x, u}[V(t+\delta, x)]
\end{aligned}
$$


By Lemma 3.4 note that $V$ is 1-Hölder continuous in $x$. We have

$$
\left|I_{\delta}^{1}\right| \leq\left[C \hat{\mathbb{E}}\left|V(t+\delta, x)-V\left(t+\delta, X_{t+\delta}^{t, x, u}\right)\right|\right]^{\frac{1}{2}} \leq\left[C \hat{\mathbb{E}}\left|X_{t+\delta}^{t, x, u}-x\right|^{2}\right]^{\frac{1}{2}} .
$$

Then by $\hat{\mathbb{E}}\left|X_{t+\delta}^{t, x, u}-x\right|^{2} \leq C \delta(C$ will change line by line $)$,

$$
\left|I_{\delta}^{1}\right| \leq C \delta^{\frac{1}{2}}
$$

According to the definition of $\mathbb{G}_{t, t+\delta}^{t, x, u}, I_{\delta}^{2}$ can be rewritten as

$$
\begin{aligned}
I_{\delta}^{2}= & V(t+\delta, x)-\hat{\mathbb{E}}\left[V(t+\delta, x)+\int_{t}^{t+\delta} f\left(s, X_{s}^{t, x, u}, Y_{s}^{t, x, u}, Z_{s}^{t, x, u}, u_{s}\right) d s\right. \\
& \left.+\int_{t}^{t+\delta} g_{i j}\left(s, X_{s}^{t, x, u}, Y_{s}^{t, x, u}, Z_{s}^{t, x, u}, u_{s}\right) d\left\langle B^{i}, B^{j}\right\rangle_{s}\right] .
\end{aligned}
$$

It yields that

$$
\begin{aligned}
\left|I_{\delta}^{2}\right| \leq & \delta^{\frac{1}{2}}\left\{\left[\hat{\mathbb{E}} \int_{t}^{t+\delta}\left|f\left(s, X_{s}^{t, x, u}, Y_{s}^{t, x, u}, Z_{s}^{t, x, u}, u_{s}\right)\right|^{2} d s\right]^{\frac{1}{2}}\right. \\
& \left.+\left[\hat{\mathbb{E}} \int_{t}^{t+\delta}\left|g_{i j}\left(s, X_{s}^{t, x, u}, Y_{s}^{t, x, u}, Z_{s}^{t, x, u}, u_{s}\right)\right|^{2} d\left\langle B^{i}, B^{j}\right\rangle_{s}\right]^{\frac{1}{2}}\right\} \\
\leq & C \delta^{\frac{1}{2}}
\end{aligned}
$$

Thus, we have

$$
V(t+\delta, x)-V(t, x) \leq C \delta^{\frac{1}{2}}
$$

This completes the proof.

\section{The viscosity solution of HJB equation}

The following theorem gives the relationship between the value function $V$ and the second-order partial differential equation (4.1).

Theorem 4.1 Let Assumptions 2.10 and 2.11 hold. $V$ is the value function defined by 2.7). Then $V$ is a viscosity solution of the following second-order partial differential equation:

$$
\begin{aligned}
& \partial_{t} V(t, x)+\sup _{u \in U} H\left(t, x, V, \partial_{x} V, \partial_{x x}^{2} V, u\right)=0, \\
& V(T, x)=\Phi(x), \quad x \in \mathbb{R}^{n},
\end{aligned}
$$

where

$$
\begin{aligned}
H(t, x, v, p, A, u) & =G(F(t, x, v, p, A, u))+\langle p, b(t, x, u)\rangle+f(t, x, v, \sigma(t, x, u) p, u), \\
F_{i j}(t, x, v, p, A, u) & =\left\langle A \sigma_{i}(t, x, u), \sigma_{j}(t, x, u)\right\rangle+2\left\langle p, h_{i j}(t, x, u)\right\rangle+2 g_{i j}(t, x, v, \sigma(t, x, u) p, u),
\end{aligned}
$$

$(t, x, v, p, A, u) \in[0, T] \times \mathbb{R}^{n} \times \mathbb{R} \times \mathbb{R}^{d} \times \mathbb{S}_{n} \times U, \sigma_{i}$ is the $i$-th column of $\sigma, G$ is defined by equation (2.1) 
For simplicity, we only consider the case $h_{i j}=g_{i j}=0$.

Suppose $\varphi \in C_{b, \text { Lip }}^{2,3}\left([t, T] \times \mathbb{R}^{n}\right)$. Define

$F_{1}(s, x, y, z, u)=\partial_{s} \varphi(s, x)+\left\langle b(s, x, u), \partial_{x} \varphi(s, x)\right\rangle+f\left(s, x, y+\varphi(s, x), z+\partial_{x} \varphi(s, x) \sigma(s, x, u), u\right)$,

$F_{2}^{i j}(s, x, u)=\frac{1}{2}\left\langle\partial_{x x}^{2} \varphi(s, x) \sigma_{i}(s, x, u), \sigma_{j}(s, x, u)\right\rangle$.

Consider the following G-BSDEs: $\forall s \in[t, t+\delta]$,

$$
\begin{aligned}
Y_{s}^{1, u}= & \int_{s}^{t+\delta} F_{1}\left(r, X_{r}^{t, x, u}, Y_{r}^{1, u}, Z_{r}^{1, u}, u_{r}\right) d r+\int_{s}^{t+\delta} F_{2}^{i j}\left(r, X_{r}^{t, x, u}, u_{r}\right) d\left\langle B^{i}, B^{j}\right\rangle_{r} \\
& -\int_{s}^{t+\delta} Z_{r}^{1, u} d B_{r}-\left(K_{t+\delta}^{1, u}-K_{s}^{1, u}\right), \\
Y_{t+\delta}^{1, u}= & 0
\end{aligned}
$$

and

$$
Y_{s}^{u}=\varphi\left(t+\delta, X_{t+\delta}^{t, x, u}\right)+\int_{s}^{t+\delta} f\left(r, X_{r}^{t, x, u}, Y_{r}^{u}, Z_{r}^{u}, u_{r}\right) d r-\int_{s}^{t+\delta} Z_{r}^{u} d B_{r}-\left(K_{t+\delta}^{u}-K_{s}^{u}\right) .
$$

Lemma $4.2 \forall s \in[t, t+\delta]$, we have

$$
Y_{s}^{1, u}=Y_{s}^{u}-\varphi\left(s, X_{s}^{t, x, u}\right)
$$

Proof. Applying Itô's formula to $\varphi\left(s, X_{s}^{t, x, u}\right)$, we have

$$
d\left(Y_{s}^{u}-\varphi\left(s, X_{s}^{t, x, u}\right)\right)=d Y_{s}^{1, u} .
$$

Since $Y_{t+\delta}^{u}-\varphi\left(t+\delta, X_{t+\delta}^{t, x, u}\right)=Y_{t+\delta}^{1, u}=0$, we obtain

$$
Y_{s}^{1, u}=Y_{s}^{u}-\varphi\left(s, X_{s}^{t, x, u}\right), \quad \forall s \in[t, t+\delta] .
$$

The proof is completed.

Consider the G-BSDE: $\forall s \in[t, t+\delta]$,

$$
Y_{s}^{2, u}=\int_{s}^{t+\delta} F_{1}\left(r, x, Y_{r}^{2, u}, Z_{r}^{2, u}, u_{r}\right) d r+\int_{s}^{t+\delta} F_{2}^{i j}\left(r, x, u_{r}\right) d\left\langle B^{i}, B^{j}\right\rangle_{r}-\int_{s}^{t+\delta} Z_{r}^{2, u} d B_{r}-\left(K_{t+\delta}^{2, u}-K_{s}^{2, u}\right)
$$

We have the following estimation.

Lemma 4.3 We have

$$
\left|\underset{u(\cdot) \in \mathcal{U}[t, T]}{\operatorname{esssup}} Y_{t}^{1, u}-\underset{u(\cdot) \in \mathcal{U}[t, T]}{\operatorname{ess} \sup } Y_{t}^{2, u}\right| \leq C \delta^{3 / 2},
$$

where $C$ is a positive constant independent of $u(\cdot)$. 
Proof. By Proposition 3.9 in [10], we have for any fixed $u(\cdot) \in \mathcal{U}[t, T]$ and $p>2$

$$
\begin{aligned}
\left|Y_{t}^{1, u}-Y_{t}^{2, u}\right|^{2} & \leq \hat{\mathbb{E}}\left[\sup _{s \in[t, t+\delta]}\left|Y_{s}^{1, u}-Y_{s}^{2, u}\right|^{2}\right] \\
& \leq C\left\{\left(\hat{\mathbb{E}}\left[\sup _{s \in[t, t+\delta]} \hat{\mathbb{E}}_{s}\left[\left(\int_{t}^{t+\delta} \hat{F}_{r} d r\right)^{p}\right]\right]\right)^{2 / p}+\hat{\mathbb{E}}\left[\sup _{s \in[t, t+\delta]} \hat{\mathbb{E}}_{s}\left[\left(\int_{t}^{t+\delta} \hat{F}_{r} d r\right)^{p}\right]\right]\right\},
\end{aligned}
$$

where $\hat{F}_{r}=\left|F_{1}\left(r, X_{r}^{t, x, u}, Y_{r}^{2, u}, Z_{r}^{2, u}, u_{r}\right)-F_{1}\left(r, x, Y_{r}^{2, u}, Z_{r}^{2, u}, u_{r}\right)\right|+\sum_{i, j=1}^{d} \mid F_{2}^{i j}\left(r, X_{r}^{t, x, u}, Y_{r}^{2, u}, Z_{r}^{2, u}, u_{r}\right)-$ $F_{2}^{i j}\left(r, x, Y_{r}^{2, u}, Z_{r}^{2, u}, u_{r}\right) \mid$. It is easy to verify that

$$
\hat{F}_{r} \leq C_{1}\left(\left|X_{r}^{t, x, u}-x\right|+\left|X_{r}^{t, x, u}-x\right|^{2}\right)
$$

where $C_{1}$ is independent of $u(\cdot)$. By standard estimates of G-SDE, we can obtain that for any $p^{\prime} \geq 2$

$$
\hat{\mathbb{E}}\left[\sup _{r \in[t, t+\delta]}\left|X_{r}^{t, x, u}-x\right|^{p^{\prime}}\right] \leq C_{2}\left(1+|x|^{p^{\prime}}\right) \delta^{p^{\prime} / 2}
$$

where $C_{2}$ is independent of $u(\cdot)$. Then by Theorem 2.13 in $[10]$ we can deduce that $\left|Y_{t}^{1, u}-Y_{t}^{2, u}\right| \leq C \delta^{3 / 2}$, where $C$ is independent of $u(\cdot)$. Thus

$$
\left|\underset{u(\cdot) \in \mathcal{U}[t, T]}{\operatorname{ess} \sup _{t}} Y_{t}^{1, u}-\operatorname{essip}_{u(\cdot) \in \mathcal{U}[t, T]} Y_{t}^{2, u}\right| \leq \operatorname{essipup}_{u(\cdot) \in \mathcal{U}[t, T]}\left|Y_{t}^{1, u}-Y_{t}^{2, u}\right| \leq C \delta^{3 / 2} .
$$

This completes the proof.

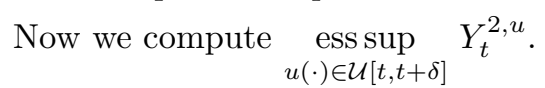

Lemma 4.4 We have

$$
\underset{u(\cdot) \in \mathcal{U}[t, t+\delta]}{e s s \sup _{t}^{2, u}}=Y_{t}^{0}
$$

where $Y^{0}$ is the solution of the following ordinary differential equation

$$
-d Y_{s}^{0}=F_{0}\left(s, x, Y_{s}^{0}, 0\right) d s, \quad Y_{t+\delta}^{0}=0, s \in[t, t+\delta]
$$

and

$$
F_{0}(s, x, y, z):=\sup _{u \in U}\left[F_{1}(s, x, y, z, u)+2 G\left(F_{2}(s, x, u)\right)\right] .
$$

Proof. By Theorem 3.7 of [11], we have

$$
Y_{s}^{2, u} \leq Y_{s}^{0}, \quad s \in[t, t+\delta]
$$

where $\left(Y^{0}, Z^{0}, K^{0}\right)$ is the solution of the following G-BSDE:

$$
Y_{s}^{0}=\int_{s}^{t+\delta} F_{0}\left(r, x, Y_{r}^{0}, Z_{r}^{0}\right) d r-\int_{s}^{t+\delta} Z_{r}^{0} d B_{r}-\left(K_{t+\delta}^{0}-K_{s}^{0}\right) \quad s \in[t, t+\delta]
$$

Since $F_{1}$ and $G\left(F_{2}\right)$ are deterministic functions, we obtain that $Z_{s}^{0}=0, K_{s}^{0}=0$ and $Y_{s}^{0}$ is the solution of equation (4.8).

We denote the class of all deterministic controls in $\mathcal{U}[t, t+\delta]$ by $\mathcal{U}_{1}$. Then, for every $u(\cdot) \in \mathcal{U}_{1}, Y^{2, u}$ is the solution of the following ordinary differential equation: 


$$
\begin{aligned}
& -d Y_{s}^{2, u}=\left[F_{1}\left(s, x, Y_{s}^{2, u}, 0, u_{s}\right)+2 G\left(F_{2}\left(s, x, u_{s}\right)\right)\right] d s, \quad s \in[t, t+\delta], \\
& Y_{t+\delta}^{2, u}=0 .
\end{aligned}
$$

It is easy to check that

$$
Y_{t}^{0}=\operatorname{ess~sup}_{u(\cdot) \in \mathcal{U}[t, t+\delta]} Y_{t}^{2, u}
$$

This completes the proof.

Finally we give the proof of Theorem 4.1 .

Proof: By Lemma 3.4, 3.6, $V$ is a continuous functions on $[0, T] \times \mathbb{R}^{n}$. We first prove that $V$ is the subsolution of (4.1).

Given $t \leq T$ and $x \in \mathbb{R}^{n}$, suppose $\varphi \in C_{b, \text { Lip }}^{2,3}\left([0, T] \times \mathbb{R}^{n}\right)$ such that $\varphi(t, x)=V(t, x)$ and $\varphi \geq V$ on $[0, T] \times \mathbb{R}^{n}$. By Theorem 3.1, we have

$$
V(t, x)=\operatorname{essup}_{u(\cdot) \in \mathcal{U}[t, t+\delta]} \mathbb{G}_{t, t+\delta}^{t, x, u ; Q}\left[V\left(t+\delta, X_{t+\delta}^{t, x, u ; Q}\right)\right]
$$

So

$$
\underset{u(\cdot) \in \mathcal{U}[t, t+\delta]}{\operatorname{ess} \sup }\left\{\mathbb{G}_{t, t+\delta}^{t, x, u}\left[\varphi\left(t+\delta, X_{t+\delta}^{t, x, u}\right)\right]-\varphi(t, x)\right\} \geq 0
$$

By (4.5), we have

$$
\operatorname{esssup}_{u(\cdot) \in \mathcal{U}[t, t+\delta]} Y_{t}^{1, u} \geq 0
$$

By (4.7) and Lemma 4.4, we get

$$
\operatorname{esssup}_{u(\cdot) \in \mathcal{U}[t, t+\delta]} Y_{t}^{2, u} \geq-C \delta^{3 / 2}
$$

and

$$
Y_{t}^{0} \geq-C \delta^{3 / 2}
$$

Thus,

$$
-C \delta^{1 / 2} \leq \delta^{-1} Y_{t}^{0}=\delta^{-1} \int_{t}^{t+\delta} F_{0}\left(r, x, Y_{r}^{0}, 0\right) d r .
$$

Letting $\delta \rightarrow 0$, we get $F_{0}(t, x, 0,0)=\sup _{u \in U}\left(F_{1}(t, x, y, z, u)+G\left(F_{2}(t, x, u)\right)\right) \geq 0$, which implies that $V$ is a subsolution of (4.1). Using the same method, we can prove $V$ is the supersolution of (4.1).

This completes the proof.

\section{Attachment}

\section{Acknowledgments}

The authors would like to thank S. Peng for many helpful discussions. 


\section{References}

[1] R. Buckdahn and J. Li, Stochastic differential games and viscosity solutions for Hamilton-JacobiBellman-Isaacs equations, SIAM J Control Optim., 47(2008), pp. 444-475.

[2] R. Buckdahna, B. Labedb, C. Rainera and L. Tamer, Existence of an optimal control for stochastic control systems with nonlinear cost functional, Stochastics, An International Journal of Probability and Stochastics Processes, 82(2010), pp. 241-256.

[3] L. Denis and C. Martini, A Theoretical Framework for the Pricing of Contingent Claims in the Presence of Model Uncertainty, The Annals of Applied Probability, 16(2006), pp. 827-852.

[4] L. Denis, M. Hu and S. Peng, Function spaces and capacity related to a sublinear expectation: application to G-Brownian motion paths, Potential Anal., 34(2011), pp. 139-161.

[5] D. Duffie and L. Epstein, Stochastic differential utility, Econometrica, 60(1992), pp. 353-394.

[6] N. El Karoui, S. Peng and M. C. Quenez, Backward stochastic differential equations in finance, Math. Finance, 7(1997), pp. 1-71.

[7] L. Epstein and S. Ji, Ambiguous Volatility, Possibility and Utility in Continuous Time, (2011), arXiv:1103.1652.

[8] L. Epstein and S. Ji, Ambiguous volatility and asset pricing in continuous time, Rev. Finan. Stud., (2013), forthcoming.

[9] W. H. Fleming, and H. M. Soner, Control Markov processes and Viscosity Solutions (New York:Springer Verlag).

[10] M. Hu, S. Ji, S. Peng and Y. Song, Backward Stochastic Differential Equations Driven by G-Brownian Motion, (2012), http://arxiv.org/pdf/1206.5889.

[11] M. Hu, S. Ji, S. Peng and Y. Song, Comparison Theorem, Feynman-Kac Formula and Girsanov Transformation for BSDEs Driven by G-Brownian Motion, (2012), arxiv.org/abs/1212.5403.

[12] M. Hu and S. Peng, On representation theorem of G-expectations and paths of G-Brownian motion, Acta Math. Appl. Sin. Engl. Ser., 25(2009), pp. 539-546.

[13] A. Matoussi, L. Piozin and D. Possamai, Second-order BSDEs with general reflection and Dynkin games under uncertainty, (2012), arXiv:1212.0476

[14] A. Matoussi, D. Possamai and C. Zhou, Robust Utility Maximization in Non-dominated Models with 2BSDE, the Uncertain Volatility Model, (2012), To appear in Mathematical Finance.

[15] A. Neufeld and M. Nutz, Superreplication under Volatility Uncertainty for Measurable Claims, (2012), preprint.

[16] M. Nutz, Random G-Expectations, (2010), To appear in Annals of Applied Probability. 
[17] M. Nutz, A Quasi-Sure Approach to the Control of Non-Markovian Stochastic Differential Equations, Electronic Journal of Probability, 17(2012), pp. 1-23.

[18] M. Nutz and J. Zhang, Optimal Stopping under Adverse Nonlinear Expectation and Related Games, (2012), preprint.

[19] E. Pardoux and S. Peng, Adapted Solutions of Backward Stochastic Equations, Systerm and Control Letters, 14(1990), pp. 55-61.

[20] S. Peng, Filtration consistent nonlinear expectations and evaluations of contingent claims, Acta Mathematicae Applicatae Sinica, 20(2004), pp. 1-24.

[21] S. Peng, Nonlinear expectations and nonlinear Markov chains, Chin. Ann. Math., 26B(2005), pp. 159184.

[22] S. Peng, G-expectation, G-Brownian Motion and Related Stochastic Calculus of Itô type, Stochastic analysis and applications, Abel Symp., 2, Springer, Berlin, (2007), pp. 541-567.

[23] S. Peng, G-Brownian Motion and Dynamic Risk Measure under Volatility Uncertainty, (2007), arXiv:0711.2834 1 .

[24] S. Peng, Multi-Dimensional G-Brownian Motion and Related Stochastic Calculus under G-Expectation, Stochastic Processes and their Applications, 118(2008), pp. 2223-2253.

[25] S. Peng, Nonlinear Expectations and Stochastic Calculus under Uncertainty, (2010), arXiv:1002.4546v1.

[26] S. Peng, Backward Stochastic Differential Equation, Nonlinear Expectation and Their Applications, in Proceedings of the International Congress of Mathematicians Hyderabad, India, 2010.

[27] S. Peng, Y. Song and J. Zhang, A Complete Representation Theorem for G-martingales, (2012), Preprint, arXiv:1201.2629v1.

[28] S. Peng, A generalized dynamic programming principle and Hamilton-Jacobi-Bellmen equation, Stochastics Stochastics Rep., 38(1992), pp. 119-134.

[29] S. Peng, Backward stochastic differential equations - stochastic optimization theory and viscosity solutions of HJB equations, in Topics on Stochastic Analysis, J. Yan, S. Peng, S. Fang, and L. Wu, eds., Science Press, Beijing, 1997, pp. 85-138 (in Chinese).

[30] T. Pham and J. Zhang, Two Person Zero-sum Game in Weak Formulation and Path Dependent Bellman-Isaacs Equation, (2012), preprint.

[31] Z. Wu and Z. Yu, Dynamic programming principle for one kind of stochastic recursive optimal control problem and Hamilton-Jacobi-Bellman equation, SIAM J.Control Optim., 47(2008), pp. 2616-2641.

[32] H. M. Soner, N. Touzi and J. Zhang, Martingale Representation Theorem under G-expectation, Stochastic Processes and their Applications, 121(2011), pp. 265-287. 
[33] H. M. Soner, N. Touzi and J. Zhang, Quasi-sure Stochastic Analysis through Aggregation, Electronic Journal of Probability, 16(2011), pp. 1844-1879.

[34] H. M. Soner, N. Touzi and J. Zhang, Wellposedness of Second Order Backward SDEs, Probability Theory and Related Fields, 153(2012), pp. 149-190.

[35] Y. Song, Some properties on G-evaluation and its applications to G-martingale decomposition, Science China Mathematics, 54(2011), pp. 287-300.

[36] Y. Song, Uniqueness of the representation for G-martingales with finite variation, Electron. J. Probab, $17(2012)$, pp. 1-15.

[37] J. Yong and X. Y. Zhou, Stochastic controls: Hamiltonian systems and HJB equations, 1999. 